\title{
Abordagem estética na Odontologia
}

\author{
Approach to esthetic dentistry \\ Enfoque en la odontología estética \\ Maria Cristina Rosifini ALVES REZENDE \\ Renato Salviato FAJARDO \\ Disciplina de Humanidade e Saúde, Departamento de Materiais Odontológicos e Prótese, \\ Faculdade de Odontologia, UNESP Univ. Estadual Paulista 16015-050, Araçatuba-SP, Brasil
}

\begin{abstract}
Resumo
O desenvolvimento e a popularização, especialmente nos últimos anos, de novas técnicas operatórias e materiais odontológicos abriram importante viés para a estetização da saúde em Odontologia, isto é, para a valorização de padrões estéticos como definidores de condições de saúde bucal, tanto por parte dos profissionais quanto por parte dos pacientes. De um modo geral as culturas ocidentais atribuem grande valor à atratividade física, influenciando assim não só a forma como as pessoas pensam e se comportam diante de outras com variadas qualidades de aparência, como também os valores culturais sobre a atratividade física, valores estes determinantes dos padrões de estética. Gênero, idade, fatores sociodemográficos e condições educacionais modulam os padrões de estética, os quais são internalizados e compõem os ideais pessoais do indivíduo, afetando diretamente, de forma positiva ou adversa, as autoavaliações e a satisfação com a própria aparência física. A beleza cria vantagem, do nascimento à vida adulta, nas relações sociais, profissionais e afetivas. Admite-se que a atração facial represente uma das mais importantes dimensões da aparência física. No entanto, as expectativas de beleza do paciente, fortemente permeadas por valores culturais e emocionais, nem sempre são clinicamente possíveis. Cabe ao profissional motivar o paciente quanto à prioridade da promoção de saúde, esclarecendo e estabelecendo expectativas realísticas no que se refere aos procedimentos cosméticos a serem realizados e seu impacto sobre a harmonia facial e qualidade de vida.
\end{abstract}

Descritores: Estética; Sorriso; Qualidade de Vida.

\begin{abstract}
The development and popularization, especially in recent years, of new surgical techniques and dental materials have opened a significant bias for aesthetics in health at dentistry, namely, to the appreciation of aesthetic standards as definers of oral health conditions, both by the professionals as by patients. Generally, western cultures place great value on physical attractiveness, thus influencing not only the way people think and behave in front of others with varied appearance qualities, but also as cultural values about physical attractiveness, which are determinants at aesthetic patterns. Gender, age, socio-demographic factors and educational conditions can modulate the aesthetic standards, which are internalized and make up the individual's personal ideals, directly affecting, positively or adversely, the self-assessments and satisfaction with one's physical appearance. Beauty provides advantage from birth to adulthood for social; professional; and affective relationships. It is assumed that the facial attraction represents one of the most important dimensions at physical appearance. Nevertheless, the patient's beauty expectations, which are strongly permeated by cultural and emotional values, are not always clinically possible. It is a professional duty motivate the patient on the priority of health promotion, clarifying and establishing realistic expectations by regarding cosmetic procedures to be performed and their impact on the facial harmony and quality of life.
\end{abstract}

Descriptors: Esthetics; Smiling; Quality of Life.

\section{Resumen}

El desarrollo y la popularización, especialmente en los últimos años, las nuevas técnicas quirúrgicas y materiales dentales han abierto un campo significativo para la estetización de la salud en odontología, que es, a la apreciación de las normas estéticas como definidores de las condiciones de salud oral, por los profesionales y los pacientes. En general, las culturas occidentales conceden un gran valor en el atractivo físico, por lo tanto influir no sólo la forma en que las personas piensan y se comportan frente a las personas con capacidades distintas apariencia, así como los valores culturales sobre el atractivo físico, determinando asi la estándares estéticos. El género, la edad, los factores sociodemográficos y condiciones educativas influencian los estándares estéticos, que son internalizadas y estabelecem los ideales personales del individuo, lo que afecta directamente, positiva o negativamente, las autoevaluaciones y la satisfacción con la apariencia física. Belleza abre ventaja desde el nacimiento hasta la edad adulta, facilitando las relaciones sociales, profesionales y afectivos. Hoy. se asume que la atracción facial representa uno de los aspectos más importantes de la apariencia física. Sin embargo, las expectativas de belleza del paciente, fuertemente impregnado por los valores culturales y emocionales no siempre son posibles clínicamente. El profesional debe motivar al paciente sobre la prioridad de promoción de la salud, aclarar y establecer expectativas realistas con respecto a los procedimientos cosméticos a realizar y su impacto en la armonía facial y la calidad de vida.

Descriptores: Estética; Sonrisa; Calidad de Vida. 


\section{INTRODUÇÃO}

$\mathrm{Na}$ atualidade, a sociedade é permeada por inúmeros discursos referentes à indústria da estética corporal $^{1,2}$. O desenvolvimento e a popularização, especialmente nos últimos anos, de novas técnicas operatórias e materiais odontológicos abriram importante viés para a estetização da saúde em Odontologia, isto é, para a valorização de padrões estéticos como definidores de condições de saúde bucal, tanto por parte dos profissionais quanto por parte dos pacientes.

Um sorriso agradável sofre influência da qualidade dos elementos dentários e dos tecidos de suporte, das relações entre dentes, maxilares e lábios durante o ato de sorrir e de sua integração harmônica na composição facial ${ }^{3}$.

Neste contexto, tanto o corpo como a cavidade bucal assumem uma posição de capital, definido por Bordieu como atributo que permite ao indivíduo o desfrutar de vantagens sociais, status ou acesso efetivo aos mecanismos de poder ${ }^{4}$.

Para Bagrichevisky e Estevão ${ }^{5}$, no processo de estetização da saúde, o corpo, ao definir e ser definido pelo meio assume um lugar de destaque no contexto de diferenciação progressiva e hierarquizada das relações humanas. Moeda de troca, graças as suas diferentes possibilidades estéticas, o corpo permite também a migração entre estratos sociais, alterando ainda trajetórias afetivas, sociais e profissionais. Housman $^{6}$ considera o fortalecimento da autoestima das pessoas fisicamente atraentes o diferencial que as leva tanto à maior socialização, quanto ao melhor desenvolvimento da assertividade e das habilidades interpessoais.

De um modo geral as culturas ocidentais atribuem grande valor à atratividade física, influenciando assim não só a forma como as pessoas pensam e se comportam diante de pessoas com variadas qualidades de aparência, como também os valores culturais sobre a atratividade física, valores estes determinantes dos padrões de estética. Gênero, idade, fatores sociodemográficos e condições educacionais modulam os padrões de estética, os quais são internalizados e compõem os ideais pessoais do indivíduo, afetando diretamente, de forma positiva ou adversa, as autoavaliações e a satisfação com a própria aparência física ${ }^{7}$.

Vale ressaltar que os parâmetros de aferição da beleza atuais sofrem imposição austera da mídia e convergem para a condição de que "ser belo" é aproximar-se de um ideal determinado de modo universal, distinto do indivíduo como ente particular e local $^{8}$.

As pessoas querem ser reconhecidas pelo outro e a Odontologia torna isso possível ${ }^{9}$ graças às opções terapêuticas e a possibilidade de resultados clínicos estéticos altamente satisfatórios ${ }^{10}$. O ativismo estético baliza a otimização do sorriso como recusa à desgraciosidade bucal, causa de desconforto psicológico, sentimento de inferioridade e até mesmo conflito emocional $^{11,12}$.

Melo et al. ${ }^{13}$ apontam que, a despeito do arsenal odontológico contar com eficientes ferramentas de análise do sorriso e referências estéticas, o sucesso do tratamento estético na Odontologia mantém estreita e vital relação com a correta interpretação das queixas estéticas dos pacientes.

Brisman $^{14}$ alerta que em muitas situações, o Cirurgião-Dentista intervém a partir da sua percepção, a qual, geralmente, difere da autopercepção do paciente, resultando em problemas não previstos na relação profissional. Nesse contexto, destaca-se a empatia e o acolhimento, uma vez que a assistência humanizada permite ao profissional conhecer as expectativas dos seus pacientes a respeito do atendimento, proporcionando ao profissional a oportunidade de adaptar a sua prática às necessidades do paciente, influenciando, assim, na melhoria da qualidade do atendimento e no melhor relacionamento entre ambos ${ }^{15-17}$.

\section{IMAGEM CORPORAL \& SORRISO \& ESTÉTICA}

A busca da beleza pelos indivíduos embute a procura da semelhança entre os pares já que a diferença poderia representar um fator de distanciamento do individuo dentro de seu próprio grupo $^{6}$.

As expressões faciais, tais como o sorriso, são essenciais na interação entre as pessoas, na manutenção da vida, na reprodução e no cuidado da prole, informando e sinalizando comportamentos, condutas e reações posteriores de quem as exibe. A literatura científica aponta o sorriso como expressão facial fortemente associada à manifestação de afeto positivo e de emoções como prazer e alegria ${ }^{18}$. Mesquita $^{19}$ acrescenta que um sorriso esteticamente agradável (dentes brancos dispostos harmonicamente) gera uma percepção positiva, o que possibilita ao indivíduo uma melhor aceitação e êxito no seu grupo social.

A estética, enquanto ciência que trata do belo e do sentimento capaz de despertar a beleza, também deve ser entendida como atributo de conforto emocional $^{20}$.

Durante a era Moderna os conceitos de belo se transformaram em subjetivos, não sendo mais atribuídos ao objeto e sim ao homem, o qual ansiaria pelo belo, iluminado pelo conhecimento sensorial entre o corpo e a mente ${ }^{21}$ estendendo-se no século XX com a razão incorporando a percepção do sensível. O culto ao padrão estético quase que inatingível, passa 
então a ter grandes dimensões, reforçando a ideia de que o padrão estético traz o prazer do pertencimento à sociedade $^{22}$.

Lipovetsky $^{23}$ lembra também que na segunda metade do século XX surge a atualidade do estético, onde tudo tende a ser compreendido e se configura como estético. A estética assim se revela em uma dimensão superficial, ou seja, a saciedade de um desejo que resulte no puro prazer. Para Veiga ${ }^{24}$, pessoas que não se sentem atraentes são mais propensas a avaliações negativas, exagerando na importância da imagem.

Ambroise Paré, no século XVI, foi quem primeiro chamou a atenção para a imagem corporal ao definir o membro fantasma como a percepção de presença de um membro ausente. Estudos posteriores de Weir Mitchell demonstraram que condições experimentais e terapêuticas seriam capazes de modificar a imagem corporal. Bonnier em 1905 descreveu a "esquematia" como distúrbio da imagem corporal, caracterizado por distorção do tamanho das áreas corpóreas. Aspectos neurológicos, fisiológicos e psicológicos permitiram a Henry Head, em 1905, a construção do conceito de esquema corporal. Este conceito descrevia a construção de um modelo ou figura de si mesmo por cada indivíduo delineando um padrão contra os julgamentos da postura e movimentos corporais ${ }^{25}$. Estudos de Schilder $^{26}$ e Shontz ${ }^{27}$ atribuíram à organização da imagem corporal um aspecto multifacetado, dentro de um contexto orgânico, social e psicológico, construído a partir de eventos diários, com forte influência de fatores emocionais.

Cash e Pruzinsky ${ }^{28}$ consideram a imagem corporal alicerçada em sete pontos:

A imagem corporal é uma experiência subjetiva e se refere às percepções, pensamentos e sentimentos sobre o corpo e suas experiências;

- $\quad$ A imagem corporal é multifacetada e passível de mudança;

As experiências da imagem corporal são permeadas por sentimentos e percepções vivenciados pelos indivíduos sobre si próprios;

- $\quad$ A imagem corporal sofre influência social ao longo de toda a vida do indivíduo;

- $\quad$ A imagem corporal não tem caráter estático: experiências corporais modificam-na constantemente;

A imagem corporal influência o processamento de informações pelo indivíduo, isto é, o seu sentir e pensar o mundo: o indivíduo vê o que espera ver;

A imagem corporal modula o comportamento do indivíduo, em particular as relações interpessoais.

\section{ESTÉTICA \& VÍNCULO}

Merhy et al. $^{29}$ lembram que, entre os profissionais da área de saúde, a busca objetiva do problema biológico tem levado a uma ação que tem como centro os procedimentos, esvaziando o interesse no paciente e empobrecendo a escuta. Deste modo, estabelecem-se ações de saúde enfraquecidas em sua dimensão cuidadora, as quais, mesmo com os avanços científicos, perdem potência e eficácia.

Silva Junior e Mascarenhas ${ }^{30}$ postulam três dimensões para o vínculo de cuidado entre o profissional e o paciente: afetividade, relação terapêutica e continuidade. A primeira dimensão, a afetividade, constrói-se a partir do profissional que gosta da sua profissão e se interessa pela pessoa do paciente. A segunda, a relação terapêutica, diz respeito à dimensão na qual o paciente é considerado sujeito no processo de tratamento e se desenvolve o sentimento de confiança entre ele e o profissional. Por fim, na dimensão continuidade, o acompanhamento do processo terapêutico é apontado como fortalecedor do vínculo e do mútuo sentimento de confiança entre profissional e paciente.

A partir do primeiro contato entre o profissional e o paciente, via de regra, já se estabelece uma linha de cuidado que permite a construção do vínculo e da ação terapêutica. Admitem-se quatro pilares de sustentação para a ação terapêutica: acolhimento, escuta, suporte e esclarecimento ${ }^{31}$.

Por meio do acolhimento, a pessoa é convidada a falar e a expor suas necessidades, indo além do simples ato do profissional recepcionar o paciente em um espaço físico específico ${ }^{32}$. Na Odontologia, ações de acolhimento são ferramentas valiosas na criação de vínculos entre o paciente e a equipe odontológica, melhorando de forma substancial a qualidade da assistência prestada ${ }^{17}$ já que auxilia no conhecimento das necessidades do paciente. $\mathrm{O}$ acolhimento gera relações humanizadas entre quem cuida e quem é cuidado, conferindo ao "cuidar" o sentido de refletir, pensar, interessar-se por, preocupar-se, considerar $^{33}$.

$\mathrm{Na}$ escuta, por sua vez, o profissional abre espaço para a catarse e produz no paciente a possibilidade de refletir sobre o que o aflige e suas causas. Ao acolher e escutar o paciente o profissional pode ainda lhe oferecer suporte, isto é, pode oferecer continência, discriminando e atendendo suas necessidades. Por fim, o vínculo de cuidado entre o profissional e paciente se assenta sobre o esclarecimento, o qual permite aumentar o arsenal de informações do paciente, estabelecendo expectativas realistas e diminuindo repercussões emocionais negativas $^{31}$.

Balint $^{34}$ enfatiza a importância da aliança terapêutica no vínculo profissional-paciente, essencial para a promoção da saúde. 


\section{DISCUSSÃO}

O corpo, na sociedade atual, vem se tornando espaço simbólico na construção dos modos de subjetividade $^{35}$.

Na Odontologia, a busca de procedimentos estéticos está fortemente atrelada ao desejo de melhorar a aparência e a autoestima, melhorando assim a qualidade de vida e o bem estar psicológico $^{36-39}$.

Vários estudos ${ }^{40,41}$ investigaram a influência do potencial psicológico na imagem do corpo entre indivíduos que procuram procedimentos estéticos. Para esses pacientes, autoimagem e autoestima são indissociáveis.

Rosemberg $^{42}$ define autoestima como o sentimento ou apreço e a consideração que uma pessoa sente por si, ou seja, o quanto se gosta, como se vê e o que pensa sobre ela mesma. Centro da vida subjetiva do indivíduo, a autoestima determina os pensamentos, sentimentos e comportamentos do indivíduo.

Castilho $^{43}$ destaca que os procedimentos estéticos são capazes de produzir aumento no bem estar psicológico do paciente por meio de mudanças na imagem corporal nos aspectos perceptivo, cognitivo, emocional e comportamental.

Schilder ${ }^{44}$ lembra que na construção da imagem corporal, isto é, na edificação do modelo pelo qual o corpo se apresenta para cada pessoa, além da história individual, pesam as relações do indivíduo com os outros.

A insatisfação do indivíduo com a imagem corporal enquanto percepções, pensamentos e sentimentos sobre o próprio corpo e experiências corporais, parece ser a primeira motivação para a busca do procedimento estético. Quando essa insatisfação é exacerbada no indivíduo pode se transformar em verdadeira aversão pela aparência, culminando em muitos casos com o transtorno da imagem corporal dismórfica (BDD), baixa autoestima, ansiedade interpessoal e má adaptação nas interações sociais. Por não se aceitar o indivíduo passa a acreditar que as outras pessoas também não o apreciam, criando em alguns casos um ciclo vicioso no qual a baixa autoestima gera a depressão a qual retroalimenta a baixa autoestima ${ }^{41}$.

Sarwer et al. $^{45}$ acredita que a procura do indivíduo por procedimentos estéticos embute a busca de resultados que repercutam positivamente para si e para os outros. No entanto, a identificação com um modelo padronizado e, geralmente, inatingível de beleza, pode acarretar o afastamento do indivíduo de sua própria identidade.

Logo, profissionais que trabalham em áreas ligadas a procedimentos estéticos, de onde emergem as queixas de insatisfação com a aparência física, devem estar aptos a auxiliar estes pacientes na busca saudável do ideal estético ${ }^{46}$.

As expectativas de beleza do paciente, fortemente permeadas por valores culturais e emocionais, nem sempre são clinicamente viáveis e diferem de indivíduo para indivíduo. Cabe ao profissional motivar o paciente quanto à prioridade da promoção de saúde sobre os resultados estéticos atingidos.

\section{CONCLUSÃO}

A beleza abre vantagem, do nascimento à vida adulta, nas relações sociais, profissionais e afetivas. A atração facial representa uma das mais importantes dimensões da aparência física.

Procedimentos estéticos na Odontologia acrescem à finalidade de devolver forma e função às estruturas bucais, o objetivo de, na harmonização do sorriso, reforçar o conforto emocional do indivíduo.

\section{REFERENCIAS}

1. Miskolci R. Corpos eletricos: do assujeitamento a estetica da existencia. Estudos Feministas. 2006; 14: 681-93.

2. Sant'anna DB. Corpos de passagem: ensaios sobre a subjetividade contemporanea. Sao Paulo: Estacao Liberdade; 2001.

3. Qualtrough AJE, Burke FJT. A look at dental esthetics Quintessence Int. 1994; 25:7-14.

4. Bourdieu P. O Poder Simbólico. Lisboa: Difel; 1989.

5. Bagrichevisky M, Estevão A. Saúde coletiva: dialogando sobre interfaces temáticas. Ilhéus: Editara da UESC; 2015.

6. Housman SB. Psychosocial Aspects of Plastic Surgery. In: McCarthy, J. G. (ed.). Plastic Surgery: General Principles. Philadelphia: W. B. Saunders Company, 1990, Vol.1, Capitulo 3, p. 113-138.

7. Sante AB. Auto-imagem e características de personalidade na busca de cirurgia plástica estética [dissertação]. Ribeirão Preto: Universidade de São Paulo; 2008.

8. Sant'anna DB. Corpos de passagem: ensaios sobre a subjetividade contemporanea. Sao Paulo: Estacao Liberdade; 2001.

9. Spear FM, Kokich VG, Mathews DP. Interdisciplinary management of anterior dental esthetics. J Am Dent Assoc. 2006;137(2):160-9.

10. Grzić R, Spalj S, Lajnert V, Glavicić S, Uhac I, Pavicić DK. Factors influencing a patient's decision to choose the type of treatment to improve dental esthetics. Vojnosanit Pregl. 2012;69(11):978-85.

11. Mondelli J, Cunha LF, Furuse AY. Remodelação cosmética para corrigir postura labial do sorriso. $\mathrm{R}$ Dental Press Estét. 2007;4(2):30-40. 
12. Feitosa DAS, Dantas DCRE, Guênes GMT, Ribeiro AIAM, Cavalcanti AL, Braz R. Percepção de pacientes e acadêmicos de odontologia sobre estética facial e dentária. RFO. 2015;14(1):23-6.

13. Melo GFB, Menezes Filho PF. Proporção áurea e sua relevância para a odontologia estética. Int J Dent. 2008;7(4):234-8.

14. Brisman AS. Esthetics: a comparison of dentist's and patients' concepts. J Am Dent Assoc 1980; 100(3):345-52.

15. Oliveira FP, Bosi MLM, Vigario PS, Vieira RS. Comportamento alimentar e imagem corporal em atletas. Rev Bras Med Esporte. 2003;9(6):348-56.

16. Guerra CT, Bertoz APM, Fajardo RS, Alves Rezende MCR. Reflexões sobre o conceito de atendimento humanizado em Odontologia. Arch Health Invest (2014);3(6):31-6.

17. Alves Rezende MCR, Lopes MRANE, Gonçalves DA, Zavanelli AC, Fajardo RS. Acolhimento e bem estar no atendimento odontológico humanizado: o papel da empatia. Arch Health Invest (2015) 4(3): 57-61.

18. Frank MG, Ekman P, Friesen WV. Behavioral markers and recognizability of the smile of enjoyment. J Pers Soc Psychol.1993;64(1):83-93.

19. Mesquita MS. O sorriso humano [dissertação]. Lisboa: Universidade de Lisboa; 2011.

20. Souza T, Held MSB. A Estética como Conforto Psicológico na Moda. VII Colóquio de Moda, 2011; Londrina. Londrina: UTFPR; 2011.

21. Hermann N. Ética, estética e alteridade. II Seminário Nacional de Filosofia e Educação Confluências Brasil, 2006; Santa Maria: UFSM; 2006.

22. Matos ALB, Silva CMS, Silva ML, Cunha J. Elaboração do vestuário para portadores de desabilidade física sob a perspectiva do design. Buenos Aires: Universidade de Palermo; 2007.

23. Lipovetsky G. O império do efêmero: A moda nas sociedades modernas. São Paulo: Editora Schwarcz; 1987.

24. Veiga AP. A institucionalização da beleza no universo feminino. IGT na Rede. 2006;3(5): Disponível em : http://www.igt.psc.br/ ojs/viewarticle.php?id=16\&layout=html

25. Barros DD. Imagem corporal: a descoberta de si mesmo. História, Ciências, Saúde - Manguinhos. 2005;12(2):547-54.

26. Schilder P. A imagem do corpo: as energias construtivas da psique. 3. ed. São Paulo: Martins Fontes; 1993.

27. Shontz, FC. Body image and Physical disability. In: Cash TF, Pruzinsky T. (ed.) Body images: development, deviance and change. New York: The Guilford Press; 1990. p. 149-68.
28. Cash TF, Pruzinsky T. Body images: development, deviance and change. New York: The Guilford Press; 1990.

29. Merhy EE.; Feuerwecker L.; Gomes MPC. Da repetição à diferença: construindo sentidos com o outro no mundo do cuidado. In: Franco, T.B. (Org.).Semiótica, afecção \& cuidado em saúde. São Paulo: Hucitec, 2010. p. 60-75.

30. Silva Júnior AG, Mascarenhas MM. Avaliação da atenção básica em saúde sob a ótica da integralidade: aspectos conceituais e metodológicos. In: Pinheiro R, Mattos RA. (Orgs.). Cuidado: as fronteiras da integralidade. Rio de Janeiro: Abrasco; 2004. p.241-57.

31. Gonçalves DA, Fiore MLM. Vínculo, acolhimento e abordagem psicossocial: a prática da integralidade. Disponível em: http://www.unasus.unifesp.br/biblioteca_virtual/es f/1/modulo_psicossocial/Unidade_16.pdf. Acesso em: 20 set.2015.

32. Teixeira RR. Humanização e atenção primária à saúde. Cienc Saude Colet. 2005;10(3):585-97.

33. Boff L. Saber Cuidar: Ética do humano: compaixão pela terra. 8 ed, São Paulo: Vozes, 2002.

34. Balint M. O médico, seu paciente e a doença. Rio de Janeiro/São Paulo, Livraria Atheneu, 1988.

35. Dantas JB. Um ensaio sobre o culto ao corpo na contemporaneidade. Rev est e pesq em psicologia. 2011;11(3). Disponível em : www.revispsi.uerj.br/ v11n3/artigos/html/v11n3a10.html. Acesso em: 20 set.2015.

36. Carminatti M, Fajardo RS, Alves Rezende MCR. Humanização do atendimento em saúde: perfil e expectativas de egressos de odontologia. Arch Health Invest 2013; 2 (Especial 2 ):134 2.

37. Capalbo LC, Carminatti M, Capalbo BC, Cury MT, Fiorin LG, Wada CM et al. Atendimento humanizado: perfil e expectativas de odontolandos. Arch Health Invest. 2014;3:(Spec Iss 3):15-6.

38. Guerra CT, Alves Rezende MCR. Humanização do atendimento em saúde : perfil dos cirurgiõesdentistas. 69f. Trabalho de Conclusão de Curso - Faculdade de Odontologia de Araçatuba, Universidade Estadual Paulista, Araçatuba, 2014.

39. Alves MCR. Aspectos psicológicos das intervenções cirúrgicas na área odontológica. Odontol Mod. 1985;12:48-52.

40. Sarwer DB, Wadden TA, Pertschuk MJ, Whitaker LA. The psychology of cosmetic surgery: a review and reconceptualization. Clin Psychol Rev. 1998;18(1): 1-22.

41. Cash TF, Pruzinsky T. Body images: development, deviance and change. New York: The Guilford Press; 1990. 
42. Rosenberg M. Society and the adolescent selfimage. Princeton: Princeton University Press; 1965.

43. Castilho, SM. A imagem corporal. Santo André: Esetec; 2001.

44. Schilder P. A imagem do corpo: as energias construtivas da psique. 3. ed. São Paulo: Martins Fontes; 1993.

45. Sarwer DB, Wadden TA, Pertschuk MJ, Whitaker LA. The psychology of cosmetic surgery: a review and reconceptualization. Clin Psychol Rev. 1998;18(1):1-22.

46. Deus LGL, Boggio RF, Carlucci AR. Influência dos transtornos psíquicos nos pacientes que procuram tratamentos estéticos. São Paulo: Instituto Brasileiro de Pesquisa e EnsinoIBRAPE; 2009.

\section{CONFLITO DE INTERESSES}

Os autores declaram não haver conflitos de interesse.

\section{AUTOR PARA CORRESPONDÊNCIA}

Maria Cristina Rosifini Alves Rezende

rezende@foa.unesp.br
Submetido em 22/01/2016 Aceito em 29/01/2016 